\title{
Evaluación Nutricional de Concentrados Proteicos de Phaseolus Iunatus y Vigna unguiculata
}

\author{
Yesid A. Marrugo-Ligardo ${ }^{(1) *}$, Piedad M. Montero-Castillo(2) y Marlene Duran-Lengua( ${ }^{(3)}$ \\ (1) Universidad de Cartagena, Facultad de Ciencias de la Salud, Barrio Zaragocilla. Carrera 50 No. 29 - 11, \\ Cartagena, Bolívar-Colombia (e-mail: timiriguaco@hotmail.com). \\ (2) Universidad de Cartagena, Facultad de Ingeniería, Programa de ingeniería de Alimentos, Avenida \\ elConsulado, Calle 30 No. 48-152. Cartagena, Bolívar-Colombia. \\ (3) Universidad de Cartagena, Facultad de Medicina, Programa de Medicina,Barrio Zaragocilla. Carrera 50 \\ No. 29 - 11, Cartagena, Bolívar-Colombia. \\ *autor a quien debe ser dirigida la correspondencia
}

Recibido Mar. 16, 2016; Aceptado May. 20, 2016; Versión final Jun. 27, 2016, Publicado Dic. 2016

\begin{abstract}
Resumen
El objetivo de este estudio fue evaluar el potencial nutricional de concentrados proteicos de Phaseolus lunatus y Vigna unguiculata. Para ello, se determinó la composición de aminoácidos, composición proximal y digestibilidad in vitro. Los resultados mostraron que a pesar de que la composición de aminoácidos determinada por HPLC fue mayor en el concentrado proteico de Vigna unguiculata; el concentrado de Phaseolus lunatus presentó características nutricionales levemente superiores, con un valor de proteína según método AOAC de 66.8\%; un cómputo químico corregido (PDCAAS) según procedimiento de $\mathrm{FAO/}$ WHO de 50.35; y un coeficiente de digestibilidad de $83.93 \%$; mientras que los valores del concentrado proteico de Vigna unguiculata, fueron de $65.29 \%$ para proteína; 41.0 para PDCAAS y de 82.11 su coeficiente de digestibilidad. Se concluye que a pesar de no encontrarse diferencias significativas en las características evaluadas de los concentrados proteicos, el concentrado Phaseolus lunatus, mostró características nutricionales levemente superiores.
\end{abstract}

Palabras clave:concentrados proteicos; potencial nutricional; calidad proteica; digestibilidad in vitro

\section{Nutritional Evaluation of Concentrated Protein of Phaseolus Iunatus and Vigna unguiculata}

\begin{abstract}
The objective of this study was the evaluation of the nutritional potential of protein concentrates of Phaseolus lunatus and Vigna unguiculata. To do this, the amino acid composition, proximate composition and in vitro digestibility were determined. The results showed that although the amino acid composition determined by HPLC was higher in the protein concentrates of Vigna unguiculata; Phaseolus lunatus concentrate presented slightly superior nutritional properties, with a value of protein according to AOAC of $66.8 \%$; a corrected chemical score (PDCAAS) according to FAO / WHO of 50.35; and a coefficient of digestibility of $83,93 \%$; while the values of protein concentrates of Vigna unguiculata, were $65.29 \%$ for protein; 41.0 for PDCAAS and 82.11 for the digestibility coefficient. It is concluded that although significant differences in the evaluated characteristics of protein concentrates were not found, Phaseolus lunatus concentrate showed slightly higher nutritional characteristics.
\end{abstract}




\section{INTRODUCCIÓN}

La seguridad nutricional es parte inseparable de la seguridad alimentaria; no es suficiente el acceso de las personas a los alimentos. Esto ha conllevado a la búsqueda de alternativas altamente nutricionales y económicas que aporten a la población una mejor alimentación (Navarro et al., 2014; Montero et al., 2015). Sin embargo, el uso de alimentos localmente disponibles está siendo impulsado como medio de diversifica-ción dietética en la lucha contra la amenaza de las deficiencias de micronutrientes, es así, como la proteína vegetal juega un papel importante en la nutrición humana, destacando el grupo de las leguminosas, las cuales proporcionan una gama de nutrientes esenciales como proteínas, hidratos de carbono de bajo índice glicémico, fibra dietética, minerales y vitaminas (Navarro et al., 2014; Valiño et al., 2015). De estas, es importante resaltar su alto contenido proteico (18-32\%), además de proporcionar una fuente de aminoácidos esenciales, péptidos bioactivos y propiedades funcionales, mejorando la estabilidad, textura y la calidad nutricional. Las leguminosas son uno de los grupos alimenticios de mayor consumo directo, por lo que se ha indicado que constituyen una de las alternativas con mayor importancia para resolver el problema de la dependencia alimentaria, pudiendo emplearse en el desarrollo de una amplia variedad de productos alimenticios (Yewande y Thomas, 2015; Vega-Macedo et al., 2014; Navarro et al., 2014; Olapade et al., 2014).

Dentro del grupo de las leguminosas que poseen semillas comestibles, destaca el frijol lima (Phaseolus lunatus), debido a sus propiedades nutritivas: contenido de proteína cercana al $29 \%$. De acuerdo a investigaciones de tipo biológico, la calidad de la proteína del frijol cocido puede ser de hasta el $70 \%$ comparada con una proteína testigo de origen animal (100\%). Teniendo en cuenta lo anterior, la leguminosa Phaseolus lunatus puede considerarse como una opción para la elaboración de productos con alto contenido de proteína, como los concentrados proteínicos (González et al., 2015; Borjórquez-Balam et al., 2013). Por su parte, el caupí (Vigna unguiculata) como miembro de la familia de las leguminosas, pertenece a Phaseoleae, la misma del frijol común (Phaseolus vulgaris). En comparación con sus parientes cercanos y muchas otras especies de cultivo, $V$. unguiculata muestra una mayor tolerancia a la sequía y tiene la capacidad para fijar nitrógeno en suelos pobres (Pan et al., 2014). Es una de las leguminosas subutilizadas que contienen cantidades adecuadas de proteínas, amino ácidos esenciales, ácidos grasos poliinsaturados, fibra dietética y minerales y vitaminas esenciales comparables con otras leguminosas comunes (González et al., 2012; Valiño et al., 2015), posee un elevado contenido de proteínas de muy buena calidad nutricional, entre 18 y 23\% (Picot et al., 2015) al igual que Phaseolus lunatus.

Pocos son los estudios enfocados a la evaluación nutricional de materias primas alimentarias de consumo masivo, las cuales podrían tener un gran potencial en la industria de alimentos, convirtiéndose en alternativas que contribuyan a mejorar la seguridad alimentaria. Cabe señalar que en un alimento, el potencial nutricional, depende en gran medida de la calidad proteica de éste, del tipo y cantidad de aminoácidos que contiene, convirtiéndose esta en un factor determinante en su valoración nutricional ya que la calidad proteica establece los aportes de nitrógeno y aminoácidos esenciales en un organismo (Marrugo et al., 2012; Cervilla et al., 2012; Valiño et al., 2015). De esta manera, la estimación del coeficiente de digestibilidad es un elemento importante para valorar este factor (Rodríguez-Miranda et al., 2014; MirandaVilla et al., 2013). De igual forma, la OMS y la FDA (Food and Drug Administration) de Estados Unidos adoptaron el cómputo químico o escore de aminoácidos corregido por la digestibilidad de la proteína en cuestión (protein digestibility corrected amino acid score -PDCAAS-) como medida para valorar la calidad de una proteína (Cervilla et al., 2012). En este orden de ideas, es importante desarrollar proyectos de investigación para la elaboración de alimentos de buena calidad nutricional, principalmente de alta calidad proteica, que permitan proyectar las leguminosas nativas como alternativas nutricionales y agroindustriales a nivel regional y nacional. En el presente estudio se evaluó el potencial nutricional de concentrados proteicos de Phaseolus lunatus y Vigna unguiculata, para valorar su posible uso en el desarrollo de nuevos productos alimenticios.

\section{MATERIALES Y MÉTODOS}

Las leguminosas se obtuvieron en los municipios de Villanueva y Turbana, departamento de Bolívar y en la central de abastos de la ciudad de Cartagena. Las semillas de Phaseolus lunatus y Vigna unguiculata secas a utilizar ( $5 \mathrm{Kg}$ de cada leguminosa) se trasladaron a la Planta piloto del Programa de Ingeniería de Alimentos de la Universidad de Cartagena, donde se adecuaron para los procesos posteriores.

\section{Diseño experimental}

En la investigación, se manejó un diseño experimental totalmente aleatorio. Las determinaciones se efectuaron por triplicado y los resultados expresados como la media \pm la desviación estándar. Se utilizó el programa PHARM / PCS versión 4, se calcularon la media y la desviación estándar de los resultados en los análisis efectuados. Para el análisis de los datos se aplicó un t-student. Se fijó el nivel de significancia a p< 0,05 . 


\section{Obtención de harinas de semillas de Phaseolus lunatus y Vigna unguiculata}

Para la obtención de las harinas, se siguió la metodología propuesta por Jaimes et al., (2014). La harina integral se obtuvo, a partir de granos enteros, realizándoseles acondicionamiento previo, posteriormente se realizó el proceso de molienda para seguidamente ser pasada por tamices de $1 \mathrm{~mm}^{2} \mathrm{~N}^{\circ} 2$ y $\mathrm{N}^{\circ} 3$, para así obtener una harina fina integral libre de impurezas.

\section{Obtención de concentrados proteicos}

Para la obtención de los concentrados proteicos se siguió el procedimiento propuesto por Jaimes et al., (2014). Se realizó método de soxhlet bajo condiciones térmicas moderadas no superiores a $40^{\circ} \mathrm{C}$, tratando de conservar las propiedades funcionales de las proteínas. Con la harina desengrasada se preparó una suspensiones de harina-agua a una relación (1:8). Para la extracción de los carbohidratos solubles y sales minerales se ajustó el pH a 8 utilizando $\mathrm{NaOH} 1 \mathrm{~N}$. Las suspensiones se mantuvieron a una temperatura de $40^{\circ} \mathrm{C}$ a 300rpm durante 30 minutos en una centrífuga. Al finalizar el período de extracción se dejaron las mezclas en reposo, se retiró el sobrenadante y el residuo amiláceo se lavó dos veces con agua, dejando cada vez 30 minutos en las mismas condiciones de temperatura $\left(40^{\circ} \mathrm{C}\right)$ y agitación (300 rpm). Los sobrenadantes de los lavados y el extracto proteico se recolectaron en el mismo recipiente beaker de 50 $\mathrm{mL}$. Una vez conseguido el extracto sé acidificó con $\mathrm{HCl} 1 \mathrm{~N}$ hasta llegar al punto de precipitación de la mayoría de las proteínas $(\mathrm{pH} 5,5)$. Alcanzado el $\mathrm{pH}$ deseado, el extracto proteico se mantuvo a temperatura de $37^{\circ} \mathrm{C}$ y luego se sometió a agitación de 300rpm durante $30 \mathrm{~min}$ en centrífuga. Posteriormente las proteínas se separaron del suero por decantación, se lavaron con agua en agitación manual constante, obteniendo el coagulo proteico el cual se sometió a decantación y secado al vacío, generándose el concentrado proteico.

\section{Determinación de las características fisicoquímicas de Phaseolus lunatus y Vigna unguiculata}

Para la determinación de las características fisicoquímicas de las harinas integrales de Phaseolus lunatus y Vigna unguiculata, y a los concentrados proteicos obtenidos a partir de esta harinas, se empleó la metodología usada por la AOAC (2003) para determinar humedad, fibra, cenizas, grasa y proteína. Una vez obtenidos estos valores se sumaron y se restaron de 100, y la diferencia se tomó como el contenido de carbohidratos (Marrugo-Ligardo et al., 2015).

\section{Determinación de aminoácidos esenciales}

Se realizó por cromatografía liquida de alta resolución (HPLC), en un cromatógrafo BAS (California, USA), con detector de fluorescencia Water 474. La detección por fluorescencia se realizó utilizando una longitud de onda de excitación de 340nm y una longitud de onda de emisión de 460nm.

\section{Digestibilidad in vitro}

Para esta determinación se aplicó el método in vitro, de acuerdo con el protocolo realizado por Pérez y Granito (2012), que consiste en someter una dispersión del concentrado, a la acción de una solución multienzimática. Las enzimas empleadas en esta determinación fueron: i) Tripsina de porcino: Type II-S, Sigma T-7409 = $23.100 \mathrm{U} / \mathrm{mL}$; ii) Quimotripsina de Bovino: Type II, Sigma C-4129 = $186 \mathrm{U} / \mathrm{mL}$ y iii) Peptidasa Intestinal Porcino: Sigma P-7010 $=0,052 \mathrm{U} / \mathrm{mL}$

\section{Determinación del cómputo químico y el corregido}

Para la determinación del cómputo químico, se aplicó el método sugerido por Cervilla et al., (2012), que consiste en dividir la cantidad de aminoácidos esenciales presentes en los concentrados proteicos entre la cantidad de aminoácidos esenciales presentes en la proteína de referencia y multiplicar este valor por cien (100). De igual forma, la determinación del cómputo químico corregido, se obtuvo aplicando el método propuesto por Cervilla et al., (2012), que consiste en multiplicar el cómputo químico por el valor de la digestibilidad.

\section{Determinación del índice de calidad proteínica}

Para calcular este valor se aplicó el método realizado por Mujica et al., (2001), que consiste en dividir el requerimiento proteico recomendado por la FAO en una edad determinada entre el requerimiento del aminoácido más limitante de la proteína en estudio. Así mismo, para calcular el requerimiento proteico recomendado por la FAO en una edad determinada, se siguió el método propuesto por Mujica et al., (2001), que consiste en dividir el requerimiento del aminoácido más limitante indicado en la proteína ideal propuesta por la $\mathrm{FAO/OMS/UNO} \mathrm{entre} \mathrm{la} \mathrm{cantidad} \mathrm{de} \mathrm{aminoácido} \mathrm{limitante} \mathrm{de} \mathrm{la} \mathrm{proteína} \mathrm{que} \mathrm{se} \mathrm{evalúa}$ 


\section{RESULTADOS Y DISCUSIÓN}

En la Tabla 1, se muestra el contenido de proteína de los concentrados de Phaseolus lunatus (C1) y Vigna Unguiculata (C2). Presentando el concentrado de Phaseolus lunatus (66,8\%) mayor contenido proteico que el de Vigna unguiculata $(65,29 \%)$. Estos valores son superiores a los reportados por Ugwuona y Suwaba, (2013) para concentrados proteicos de Canavalia ensiformis (49,47\%). Aunque en este mismo sentido presentaron menores valores proteicos que los reportados por Jaimes et al., (2014) y Ghribi et al., (2015) para concentrados de Prosopis juliflora (75\%) y Cicer arietinum L. (78,53\%) respectivamente.

Tabla 1: Composición proximal de Concentrados proteicos de Phaseolus lunatus (C1) y Vigna unguiculata (C2)

\begin{tabular}{|l|c|c|}
\hline Parámetros & C1 & C2 \\
\hline Fibra (\%) & $1,87 \pm 0,38$ & $1,85 \pm 0,14$ \\
\hline Cenizas (\%) & $4,4 \pm 0,04$ & $4,56 \pm 0,07$ \\
\hline Proteínas (\%) & $66,8 \pm 0,29$ & $65,29 \pm 0,35$ \\
\hline Humedad (\%) & $0,89 \pm 0,39$ & $1,2 \pm 0,33$ \\
\hline Grasa (\%) & $0,76 \pm 0,08$ & $0,78 \pm 0,07$ \\
\hline Carbohidratos (\%) & $25,28 \pm 1,18$ & $26,32 \pm 0,96$ \\
\hline
\end{tabular}

En lo referente al contenido de fibra, el concentrado de C1, mostró mayor valor (1,87\%) que C2 (1,85\%). Estos resultados son semejantes a los presentados por Domínguez (2010) y Betancur-Ancona et al., (2004), quienes reportaron 1,83\% y 1,82\% respectivamente para Phaseolus lunatus. De igual forma Jaimes et al., (2014) reportaron valores similares para concentrado de Prosopis juliflora (1,90\%). Por su parte, los resultados de Ugwuona y Suwaba (2013) para concentrados de Canavalia ensiformes fueron mayores para este atributo.

El contenido graso de los concentrados proteicos en estudio presentan valores de $0,76 \%$ para $\mathrm{C} 1$ y $0,78 \%$ para C2, valores muy inferiores a 3,12\% y $4.5 \%$ reportados por Domínguez (2010) para concentrado de Phaseolus lunatus y por Ghribi et al., (2015) para Cicer arietinum L. respectivamente. Por su parte Jaimes et al., (2014) y Ugwuona y Suwaba (2013) presentaron 0,77\% para Prosopis juliflora y 0,15\% para Canavalia ensiformes, valores semejante a los obtenidos en este estudio. Phaseolus lunatus y Vigna unguiculta en cuanto al contenido de carbohidratos, se comportaron como la mayoría de los concentrados proteicos obtenidos de otras leguminosas, mostrando valores superiores al $20 \%$ y menores a $30 \%$; esto es $25,28 \%$ para C1 y 26,32\% C2, como lo obtenido por Jaimes et al., (2014) para Prosopis juliflora (25,3\%)

En la Tabla 2, se muestran los valores de digestibilidad proteica de las dos leguminosas estudiadas junto con el de la proteína patrón. También se presenta la proteína bruta de cada variedad y el porcentaje de proteína disponible que se deriva de la digestibilidad encontrada. De los concentrados proteicos evaluados el de Phaseolus lunatus, presentó el mayor valor de digestibilidad $(83,93 \%)$ y mayor valor de proteína disponible a pesar de que tiene un valor inferior de proteína bruta (22,45\%). Los valores de proteína disponibles expresan el contenido de proteína bruta corregido por digestibilidad (Ver Tabla 2).

Los resultados encontrados, en cuanto a digestibilidad de los dos concentrados, en estudio, son levemente superiores a los reportados por Pérez y Granito (2012) para concentrado proteico obtenido de granos crudos de Cajanus cajan $(81,6 \%)$ e inferior al valor del concentrado de Phaseolus vulgaris $(90,1 \%)$. En ese mismo sentido, la digestibilidad proteica in vitro reportada para los concentrados proteicos, tienen niveles superiores a los reportados para la leguminosa Canavalia ensiformis (57,52\%) por Zamora (2003), en un estudio sobre recursos alimenticios alternativos con gran potencial para la alimentación animal y humana.

Tabla 2: Digestibilidad "in vitro" de la proteína presente en los concentrados proteicos de Phaseolus lunatus y Vigna unguiculata

\begin{tabular}{|l|c|c|c|}
\hline \multicolumn{1}{|c|}{ Muestra } & $\begin{array}{c}\text { Coeficiente de } \\
\text { Digestibilidad }\end{array}$ & $\begin{array}{c}\text { Proteína Bruta } \\
(\%)\end{array}$ & $\begin{array}{c}\text { Proteína } \\
\text { Disponible (\%) }\end{array}$ \\
\hline $\begin{array}{l}\text { Concentrado Proteico de } \\
\text { Phaseolus lunatus }\end{array}$ & $83,93 \pm 2,6$ & 22,45 & 18,84 \\
\hline $\begin{array}{l}\text { Concentrado Proteico de } \\
\text { Vigna Unguiculata }\end{array}$ & $82,11 \pm 2,8$ & 22,63 & 18,58 \\
\hline Casilan(sustancia patrón) & 92,7 & - & - \\
\hline
\end{tabular}


La Tabla 3, muestra el perfil de aminoácidos de los concentrados proteicos de Phaseolus lunatus y Vigna unguiculata y los requerimientos de aminoácidos de niños de un año como proteína ideal según la FAO/OMS (1985). El perfil de aminoácidos de los concentrados proteicos, muestra que Vigna unguiculata desde el referente de composición de aminoácidos tiene proteína de mejor calidad proteica que Phaseolus lunatus aunque sin diferencia significativa. De igual forma muestra también que los concentrados proteicos de Phaseolus lunatus y Vigna unguiculata, conservan la tendencia de la mayoría de las leguminosas, siendo deficientes en aminoácidos azufrados (metionina y cisteína), Phaseolus lunatus presentó un valor de 1,4 y Vigna unguiculata de $1,3 \mathrm{~g} / 100 \mathrm{~g}$ de proteína; estos valores son deficientes si los comparamos con la proteína patrón de la FAO/OMS/UNU que presenta un valor $2,5 \mathrm{~g} / 100 \mathrm{~g}$ de proteína.

Tabla 3: Perfil de aminoácidos de los concentrados proteicos de Phaseolus lunatus y Vigna unguiculata y los requerimientos de aminoácidos de niños de un año como proteína ideal según la FAO/OMS (1985).

\begin{tabular}{|l|c|c|c|c|c|}
\hline $\begin{array}{l}\text { Aminoácidos } \\
\text { Esenciales }\end{array}$ & $\begin{array}{c}\text { Concentrado proteico } \\
\text { de Phaseolus } \\
\text { lunatus. }\end{array}$ & $\begin{array}{c}\text { Concentrado } \\
\text { proteico de } \\
\text { V. unguiculata }\end{array}$ & $\begin{array}{c}\mathrm{mg} / \mathrm{g} \text { de proteína. } \\
\text { Patrón } \\
\text { FAO/OMS/UNU }\end{array}$ & $\begin{array}{c}\text { Computo } \\
\text { químico } \\
\text { P. lunatus }\end{array}$ & $\begin{array}{c}\text { Computo } \\
\text { Químico } \\
\text { V. unguiculata }\end{array}$ \\
\hline Isoleucina & $4,2 \pm 2,3$ & $4,8 \pm 0,34$ & 2,8 & 1,5 & 1,7 \\
\hline Leucina & $8,0 \pm 0,76$ & $8,6 \pm 0,67$ & 6,6 & 1,2 & 1,3 \\
\hline Lisina & $5,3 \pm 1,8$ & $8,4 \pm 0,78$ & 5,8 & 0,9 & 1,4 \\
\hline Metionina+cisteína & $1,4 \pm 0,9$ & $1,3 \pm 0,89$ & 2,5 & 0,6 & 0,5 \\
\hline Fenilalanina +tirosina & $9,2 \pm 0,87$ & $9,7 \pm 0,45$ & 6,3 & 1,4 & 1,5 \\
\hline Treonina & $3,6 \pm 0,85$ & $4,2 \pm 0,9$ & 3,4 & 1,0 & 1,2 \\
\hline Triptófano & $0,7 \pm 1,6$ & $0,7 \pm 1,8$ & 1,1 & 0,6 & 0,6 \\
\hline Valina & $4,1 \pm 0,7$ & $5,8 \pm 0,53$ & 3,5 & 1,1 & 1,6 \\
\hline
\end{tabular}

Lo anterior concuerda con el estudio realizado por Betancur-Ancona et al., (2004) donde los azufrados presentan valores también deficientes de $1,7 \mathrm{~g}$ y $1,2 \mathrm{~g} / 100 \mathrm{~g}$ de proteína para phaseolus lunatus y Vigna unguiculata respectivamente. El resto de aminoácidos esenciales; isoleucina, leucina, lisina, fenilalanina+tirosina, treonina y valina se encuentran en mayor cantidad en el concentrado Vigna unguiculata contra Phaseolus lunatus. Pero ambos concentrados presentan valores superiores que la proteína patrón para los aminoácidos en mención.

En la Tabla 4 se presenta la composición de aminoácidos de los concentrados proteicos en estudio junto con las necesidades de aminoácidos en diferentes grupos de edad según FAO/OMS/UNU y se observa que los concentrados proteicos de Phaseolus lunatus y Vigna unguiculata satisfacen las necesidades de aminoácidos en un adulto excepto en los aminoácidos azufrados. El concentrado proteico de Vigna unguiculata satisface las necesidades de aminoácidos esenciales de pre-escolares, escolares y adultos excepto en los aminoácidos azufrados y triptófano. Este mismo concentrado no satisface las necesidades de aminoácidos esenciales de los lactantes debido a que tiene menor cantidad en los aminoácidos azufrados (13), leucina (86) y triptófano (7) en tanto que las necesidades de los lactantes en estos mismos aminoácidos son de 42,93 y 17 respectivamente.

Tabla 4: Composición de aminoácidos de concentrados proteicos de Phaseolus lunatus y Vigna unguiculata y necesidades de aminoácidos en diferentes grupos de edad según FAO/OMS/UNU

\begin{tabular}{|l|c|c|c|c|c|c|c|c|}
\hline $\begin{array}{c}\text { Aminoácidos } \\
\text { (mg/g de proteínas } \\
\text { crudas) }\end{array}$ & Lactantes & $\begin{array}{c}\text { Pre- } \\
\text { escolares } \\
(2-5 \text { años) }\end{array}$ & $\begin{array}{c}\text { Escolares } \\
(10-12 \text { años })\end{array}$ & Adultos & C1 P. lunatus & $\begin{array}{c}\text { C2 V. } \\
\text { unguiculata }\end{array}$ & $\begin{array}{c}\text { Puntaje } \\
\text { Químico } \\
\text { C1 }\end{array}$ & $\begin{array}{c}\text { Puntaje } \\
\text { Químico } \\
\text { C2 }\end{array}$ \\
\hline Histidina & 26 & 19 & 19 & 16 & 19 & 35 & 1,00 & 0,54 \\
\hline Isoleucina & 46 & 28 & 28 & 13 & 42 & 48 & 0,66 & 0,58 \\
\hline Leucina & 93 & 66 & 44 & 19 & 80 & 86 & 0,82 & 0,76 \\
\hline Lisina & 66 & 58 & 44 & 16 & 53 & 84 & 1,09 & 0,69 \\
\hline Metionina + Cistina & 42 & 25 & 22 & 17 & 14 & 13 & 1,78 & 1,92 \\
\hline $\begin{array}{l}\text { Fenilalanina + } \\
\text { Tirosina }\end{array}$ & 72 & 63 & 22 & 19 & 92 & 97 & 0,68 & 0,64 \\
\hline Treonina & 43 & 34 & 28 & 9 & 36 & 42 & 0,94 & 0,80 \\
\hline Triptófano & 17 & 11 & 9 & 5 & 7 & 7 & 1,57 & 1,57 \\
\hline Valina & 46 & 35 & 25 & 13 & 41 & 58 & 0,85 & 0,60 \\
\hline $\begin{array}{l}\text { Total incluida } \\
\text { histidina }\end{array}$ & 434 & 320 & 222 & 111 & 365 & 435 & - & - \\
\hline $\begin{array}{l}\text { Total excluida } \\
\text { histidina }\end{array}$ & 339 & 241 & 127 & 384 & 470 & - \\
\hline
\end{tabular}


Por otra parte, el concentrado proteico de Phaseolus lunatus satisface las necesidades de aminoácidos esenciales sólo de escolares y adultos; a excepción de los requerimientos de triptófano y aminoácidos azufrados en este grupo de edades (Ver Tabla 4). En cuanto a los lactantes, el concentrado de Phaseolus lunatus, es deficiente en siete (7) de los aminoácidos esenciales que requieren, solo satisface las necesidades de los aminoácidos aromáticos. De modo similar, este concentrado proteico no satisface por completo las necesidades de aminoácidos en los pre-escolares, puesto que presenta deficiencia en tres (3) de los aminoácidos esenciales. Sin embargo, al calcular el índice de calidad proteínica de los concentrados en estudio para niños en edad pre-escolar, se observó que la proteína del concentrado de Phaseolus lunatus obtiene un mayor índice de calidad proteínica (62\%), comparado con la proteína del concentrado de Vigna unguiculata (57\%).

\section{CONCLUSIONES}

Analizados los datos obtenidos en la fase experimental de esta investigación, se puede concluir que las leguminosas Phaseolus lunatus y Vigna unguiculata, tienen gran similitud en lo referente al potencial nutricional. Por lo que, estos concentrados proteicos gracias a las características nutricionales que poseen: contenido de proteína, coeficiente de digestibilidad, proteína disponible, cómputo químico e índice de calidad proteica, brindan la posibilidad de ser incorporados a ciertos tipos de alimento para enriquecerlos nutricionalmente y mejorar su calidad nutritiva.

\section{AGRADECIMIENTOS}

Los autores agradecen a las autoridades de la Universidad de Cartagena y especialmente, a la Facultad de Ciencias Químicas y Farmacéuticas por facilitar sus instalaciones para la ejecución de este trabajo.

\section{REFERENCIAS}

AOAC., Métodos de análisis de la Asociación Oficial de Química Analítica para determinar humedad, fibra, cenizas, grasa y proteína, Cap. 32: 1, 2, 5 y 14, Washington, U.S.A. (2003)

Betancur-Ancona, D., S. Gallegos-Tintoré y L. Chel-Guerrero, Wet-fractionation of Phaseolus lunatus seeds: partial characterization of starch and protein, doi: 10.1002/jsfa.1804, Journal of the Science of Food and Agriculture, 84(10), 1193-1201 (2004)

Borjórquez-Balam, E. y otros cuatro autores, Evaluación de la capacidad antimicrobiana de fracciones peptídicas de hidrolizados proteínicos de frijol lima (Phaseolus lunatus), En Segura M., L. Chel y D. Betancur (Eds.), Bioactividad de péptidos derivados de proteínas alimentarias, OmniaScience, pp 139-154, Barcelona, España (2013)

Cervilla, N.S. y otros tres autores, Determinación del contenido de aminoácidos en harinas de quinoa de origen argentino. Evaluación de su calidad proteica, Actualización en nutrición, 13(2), 107-113 (2012)

Domínguez, M.M., Aislamiento de biopeptidos con actividad inhibitoria de la enzima convertidora de angiotensina-1 a partir de hidrolizados de P. Lunatus, Tesis Doctoral, Dpto Ciencias Médico Biológicas, Instituto Politécnico Nacional, México D.F (2010)

FAO,OMS,UNU., Necesidades de energía y de proteínas, Informe de una Reunión Consultiva Conjunta FAO/OMS/UNU de Expertos, Serie de Informes Técnicos, No.724, 56-68, OMS, Ginebra (1985)

Ghribi, A. M. y otros nueve autores, Purification and identification of novel antioxidant peptides from enzymatic hydrolysate of chickpea (Cicer arietinum L.) protein concentrate, doi: 10.1016/j.jff.2014.12.011, Journal of functional foods, 12, 516-525 (2015)

González, F. J. J., S. H. Maldonado, y M. G. Hernández, Atributo nutricional y nutracéutica de panqué y barritas a base de harina de frijol (Phaseolus vulgaris L.), doi: http://dx.doi.org/10.18633/bt.v17i3.231, Revista de Ciencias Biológicas y de la Salud, 17(3), 9-14 (2015)

González, L.A. y otros tres autores, Evaluación de la ensilabilidad in vitro de granos de canavalia (Canavalia ensiformis) y vigna (Vigna unguiculata), solos o mezclados con granos de sorgo (Sorghum bicolor), Revista Cubana de Ciencia Agrícola, 46(1), 55-62 (2012)

Jaimes, M. J., D. Restrepo y D. Acevedo, Preparation and determination of the functional properties of the trupillo proteic concentrated (Prosopis juliflora), Biotecnología en el Sector Agropecuario y Agroindustrial, 12(1), 144-152 (2014) 
Marrugo, L. Y. A., P.M. Montero, y M. Duran, Propiedades funcionales de concentrados proteicos de Phaseolus lunatus y Vigna unguiculata, Vitae, 19(1), S403-S405 (2012)

Marrugo-Ligardo, Y. A. y otros tres autores, Factibilidad de almacenamiento de semillas de ajonjolí (sesamum indicum L.) en bolsas silobag, Biotecnología en el Sector Agropecuario y Agroindustrial, 13(1), 83-89 (2015)

Miranda-Villa, P. P., Y. A. Marrugo-Ligardo, y P. M. Montero-Catillo, Caracterización Funcional del Almidón de Fríjol Zaragoza (Phaseolus Lunatus L.) y Cuantificación de su Almidón Resistente, Revista Tecno Lógicas, (30), 17-32 (2013)

Montero, C. P. M., Y. A. Marrugo, y L. C. Julio, Protein Quality of Rice Drinks Fortified with Bovine and Porcine Blood Plasma, doi: http://dx.doi.org/10.15446/rfnam.v68n1.47835, Revista Facultad Nacional de Agronomía, Medellín, 68(1), 7487-7496 (2015)

Mujica, A., Jacobsen, S. E., Izquierdo, J. y Marathee, J., Quinua (Chenopodium quinoa Willd). Ancestral cultivo andino, alimento del presente y futuro, CONDESAN, Cap. 3: 246-279, Santiago, Chile (2001)

Navarro, V. C. L., D. Restrepo y J. Pérez, El guandul (Cajanus cajan) una alternativa en la industria de los alimentos, Biotecnología en el Sector Agropecuario y Agroindustrial, 12(2), 197-206 (2014).

Olapade, A. A., Y. O. Babalola, y O. C. Aworh, Quality attributes of fufu (fermented cassava) flour supplemented with bambara flour, International Food Research Journal, 21(5), 2025-2032 (2014)

Pan, L. y otros cinco autores, Development of 12 chloroplast microsatellite markers in Vigna unguiculata (Fabaceae) and amplification in Phaseolus vulgaris, doi: 10.3732 / apps.1300075, Applications in plant sciences, 2(3) (2014)

Pérez, S. y M. Granito, Concentrados de Cajanus cajanus y Phaseolus vulgaris fermentados e hirolizados: ingredientes funcionales para el desarrollo de alimentos, Interciencia, 37(6), 432-437 (2012)

Picot, J. A. y otros cuatro autores, Modificación de variables productivas por inclusión del "poroto caupí"( Vigna unguiculata) en la alimentación de cerdos, Revista veterinaria, 26(1), 49-53 (2015)

Rodríguez-Miranda, J. y otros siete autores, Efecto de la concentración de harina de frijol (Phaseolus vulgaris L.), contenido de humedad y temperatura de extrusión sobre las propiedades funcionales de alimentos acuícolas, Revista Mexicana de Ingeniería Química, 13(3), 469-663 (2014)

Suárez, L. M. M., A. Kizlansky y L.B. López, Evaluación de la calidad de las proteínas en los alimentos calculando el escore de aminoácidos corregido por digestibilidad, Nutrición hospitalaria: 21(1), 47-51(2006)

Ugwuona, F. U., y S. Suwaba, Effects of Defatted Jack Bean Flour and Jack Bean Protein Concentrate on Physicochemical and Sensory Properties of Bread, doi: 10.1016 / S0189-7241 (15) 30073-4, Nigerian Food Journal, 31(2), 25-32 (2013)

Valiño, E. y otros cuatro autores, Mejora del valor nutritivo de las leguminosas temporales Vigna unguiculata, Canavalia ensiformis, Stizolobium niveum, Lablab purpureus mediante el procesamiento de sus granos con Trichoderma viride M5-2, http://4www.redalyc.org/articulo.oa?id=193036208014, ISSN: 00347485, Revista Cubana de Ciencia Agrícola, 49(1), 81-89 (2015)

Vega-Macedo, M. y otros cuatro autores, Inseguridad alimentaria y variedad de la alimentación en hogares mexicanos con niños menores de cinco años,

http://www.scielo.org.mx/scielo.php?script=sci_arttext\&pid=S0036-36342014000700005, ISSN: 0036-3634, Salud Pública de México, 56 (1), s21-s30 (2014)

Yewande, B.A., y A.O. Thomas, Effects of processing methods on nutritive values of Ekuru from two cultivars of beans (Vigna unguiculata and Vigna angustifoliata), doi: 10.5897/AJB2014.14393, African Journal of Biotechnology, 14(21), 1790-1795 (2015).

Zamora, N.C., Efecto de la extrusión sobre la actividad de factores antinutricionales y digestibilidad in vitro de proteínas y almidón en harinas de Canavalia ensiformis, Archivos Latinoamericanos de Nutrición, 53(3), 293-298 (2003) 
